\title{
Fault level prediction for distribution network using fuzzy logic identifier
}

\author{
Fang Shi ${ }^{1}$ and Bin Wang ${ }^{2}$ \\ ${ }^{1}$ Key Laboratory of Power System Intelligent Dispatch and Control of the Ministry of Education, Jinan 250061, China \\ ${ }^{2}$ Shanghai Normal University, Shanghai 200234, China
}

\begin{abstract}
With the increasing penetration of the renewable power energy sources, the potential fault current of the distribution power systems changes more frequently as the connection structure of the distribution power system varies from time to time. Traditionally, the fault level can be estimated through short circuit analysis which is time consuming and sometimes difficult as it needs to know the parameters of the transmission line and transformers as well as the structure of the power system. In this paper, an online-used fault level prediction method is proposed via monitoring the phasor value of the local positive-sequence voltage and current. The ratio of the voltage change and current change are used to distinguish the natural deviation of the load from the switching operations or disturbances on the grid side. Several continuous changes of the voltage and current caused by load fluctuations are recorded and used to parameterize the equivalent circuit of the power system and to estimate the fault current level. A fuzzy logic identifier is used for adaptively selecting and recording the satisfactory changes by defining an index of confidence level. The implementation of the proposed scheme is demonstrated in a relay after introducing a low-voltage blocking function. A typical distribution power system with renewable generators is established in PSCAD/EMTDC and is used to verify the effectiveness and accuracy of the proposed method under various load changing conditions.
\end{abstract}

\section{Introduction}

Circuit breakers are of great importance to the safety of the transmission and distribution power system, which will disconnect the fault component or the electricity supply affected sections of the grid in case of a fault. These circuit breakers must be chosen with a high enough capacity to break the possible flow of current even under the worst fault conditions [1]. The network infrastructure also needs to be able to withstand the current flow during the period before the circuit breakers isolate the affected network to prevent destructive failure. Therefore, the fault level prediction (FLP) is needed both for the planning and operation of a power system. Then the satisfying circuit breaker is selected from the available product list. Normally, the fault level is calculated by investigating the parameters of the transmission line and transformers in the power system and there will be a margin for the breaking capacity so the breakers can have the ability to cope with the increase of the fault level in a near future [2].

However, with the increasing penetration of distribution generators (DG), such as the wind power plants and the photovoltaic (PV) power plants, the fault level or fault current of the distribution power grid changes dramatically more often than ever before [3]. Sometimes the extreme fault current will exceeds the capacity of the breakers and lead to switching off failure which will threat the safe operation of the power system. Nevertheless, the settings of the protection relays also need to be changed according to the new operation conditions [4]. For example, if a big proportion of the supplying power source changes from traditional generators into PV plants, the fault current will decrease as the convertors' contributions to the fault current is negligible. Under this circumstance, the previous setting value of the protection relays could be invalid for disconnecting short circuit fault, which may lead to overheating problems for the devices, such as transmission lines and transformers. Therefore, an online used FLP is required to predict the real-time fault level of the power system.

Traditionally, the fault current can be calculated by utilities in two ways, or a combination of both: (1) Using empirical principles to calculate the fault level by summing the known contributions from the major elements in the power grid, i.e., transformers or generators; (2) Creating an analytical parameterized model of the power grid section that is closely related to the fault level and compute the result mathematically. Both the methods are valid for the FLP during the power system planning period. The rough model can be used and a conservative value can be acquired using the first method, while the latter method can provide an accurate result but is complex and time consuming, so it has been used mainly at higher network voltages (for example at $33 \mathrm{kV}$ and above) $[5,6]$.

In the last two decades, some model free methods have been proposed to predict the fault level of a radial network or a radialized section of an interconnected 
network using the measurements of the local voltage and current [7-9]. The impedance of the power-supply network is evaluated from the naturally occurring disturbances in [10], then the fault level is calculated. The results are encouraging and the method can be used online for continuous assessment of the fault level. Some portable device can be expediently used for calculating the fault level by connecting to the terminal of the breaker and measuring the voltage and current change induced by the deviation of the loads or by artificial disturbances [11]. A predictive fault level algorithm is proposed using the locally measured voltage and current in a protection relays by distinguishing the disturbance on the load side from on the grid side in [12]. However, only the sudden load change is used and a fixed threshold value is set for identifying the change of the voltage or the current. For the slow load changing scenarios, the method could be invalid for the power grid.

There are also some other methods can be used for calculating the equivalent impedance of the power system as well as the fault level in a more active way. In [5], the artificial disturbances are applied to the power system and the change of the voltage and current are used for calculating the impedance. In [13], the harmonic current with a frequency band around the fundamental frequency is injected to the power system and then the impedance and fault current are estimated. Some hybrid techniques use both the artificial (or controlled) disturbances and natural disturbances to measure the voltage and current change and to predict the fault level [14]. The disadvantages for these methods are obvious as extra hardware investment is needed.

In this paper, an adaptive fuzzy logic identifier is designed to detect the feasible load fluctuations for estimating the fault level. The change of the voltage and current are recorded and monitored simultaneously for a specified time duration. Three quantized variables, ie., the change of the voltage, the change of the current and the calculated equivalent impedance, are analyzed and the weighted summation of the three states is used as the index to identify the desired fluctuation occurs on the load side. Then the fault level is calculated when the fluctuation is obvious enough and a confidential level of the result is given at the same time.

\section{Basic model and problem formulation}

Thevenin Equivalent circuit can be used for calculating the fault current level when only local voltage and current is available. To make the paper self-contained, the basic theory is illustrated as following. The details can also be found in [12].

Take one distribution power network with DGs (in Fig. 1) as one example. Suppose the fault level before the breaker CB2 needs to be determined. The power system upstream the breaker can be represented using the equivalent circuit shown in Fig. 2 by Thevenin's theory, where $E_{e q}$ and $Z_{e q}$ are the inner voltage and the impedance of the equivalent voltage source, respectively. If $E_{e q}$ and $Z_{e q}$ are known, then the fault level can be simply predicted as:

$$
I_{F L}=E_{e q} / Z_{e q}
$$

So the key point is to calculate $\mathrm{E}_{\mathrm{eq}}$ and $\mathrm{Z}_{\mathrm{eq}}$.

Suppose $E_{e q}$ and $Z_{e q}$ are constant and $Z_{L}$ changes from $Z_{L 1}$ to $Z_{L 2}$, which is based on the following two assumptions: 1) There is no major changes on the structure of the power grid upstream of CB2; 2) Only the load downstream of $\mathrm{CB} 2$ changes significantly so as the voltage and current at CB2 change from $U_{1}$ and $I_{1}$ to $U_{2}$ and $I_{2}$ accordingly. Normally, there will be some large capacity synchronous load connected to the feeder, such as production line of factories or large electric motors. If these loads trip on/off or fluctuate in a short time during which there is no changes or only trivial alteration on the structure and operation states on the grid side, then the above assumptions are justifiable.

Consequently, the following equations will hold (the directions of the current and voltage are as indicated in Fig. 2):

$$
\begin{aligned}
& \dot{E}_{e q}=\dot{U}_{1}+\dot{I}_{1} Z_{e q} \\
& \dot{E}_{e q}=\dot{U}_{2}+\dot{I}_{2} Z_{e q}
\end{aligned}
$$

This equation set is solvable if $U_{1}$ and $I_{1}$ are different from $U_{2}$ and $I_{2}$. Essentially, it is a parameter identification problem for the linear circuit model. Two different states are enough to estimate $E_{e q}$ and $Z_{e q}$ but more different states would yield an accurate result.But if the difference is trivial, the errors could be quite large because of the inexactitude in the measurement, analogdigital conversion transition as well as the numerical calculation.

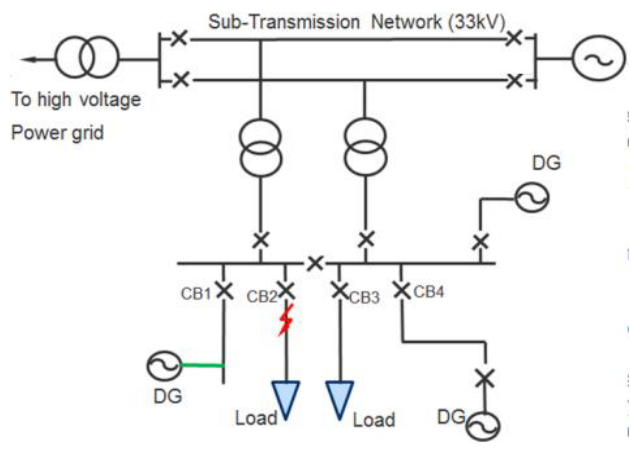

Figure 1. Example of a distribution network.

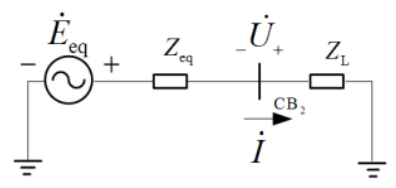

Figure 2. The equivalent circuit for fault level caculation.

However, in the practical power systems, the change of the voltage and current can also be caused by the disturbance occurs on the grid side (before $\mathrm{CB}_{2}$ in Fig. 2). Under this circumstance, $Z_{e q}$ will change in the above two equations. Consequently, we have to wait until the next load fluctuation occurs to form the above two equations with a new value of $Z_{e q}$. Therefore, it is critical to identify the disturbance occur on the grid side or on the load side. 
When the disturbance occurs on the load side, from the above two equations, it can be written as:

$$
Z_{e q}=-\frac{\dot{U}_{2}-\dot{U}_{1}}{\dot{I}_{2}-\dot{I}_{1}}=-\frac{\Delta \dot{U}}{\Delta \dot{I}}
$$

On the contrary, when the disturbance occurs on the grid side, ie., $Z_{L}$ is constant and $E_{e q}$ and $Z_{e q}$ are changeable, we can have the following two equations using the Kirchhoff's Voltage Law (KVL) on the circuit from the breaker to the downstream load:

$$
\begin{aligned}
& 0=\dot{U}_{1}-\dot{I}_{1} Z_{L} \\
& 0=\dot{U}_{2}-\dot{I}_{2} Z_{L}
\end{aligned}
$$

So we have:

$$
Z_{L}=\frac{\dot{U}_{2}-\dot{U}_{1}}{\dot{I}_{2}-\dot{I}_{1}}=\frac{\Delta \dot{U}}{\Delta \dot{I}}
$$

For the practical power system, the real part of $Z_{e q}$ and $Z_{L}$ will be greater than zero, which implies that the transfer conductance of the power grid is not ignored and there exits active load on the feeder, respectively. Then for a set of directional unknown voltage and current change, the following index can be used to distinguish whether the disturbance occurs on the grid side or on the load side:

$$
\operatorname{Dir}=-\operatorname{real}\left(\frac{\Delta \dot{U}}{\Delta \dot{I}}\right)
$$

Specifically, when Dir $>0$, it means the disturbance or fluctuation occur on the load side; when Dir $<0$, the disturbance or fluctuation will be seen as occurring on the grid side.

The above conculstion is based on the assumption that the disturbance never occur simultaneously both on grid side and on load side. It could happen (practically and legally) but the chances are slim as the monitoring devices can distinguish the two consistive changes in a very short time, for example, two cycles (40ms). Even they did happen exactly at the same time, this kind of disturbance can be ignored and only the desired flucuation on the load side is used to calculate the fault level.

So there are three requirements for an accurate FLP using the above mentioned method. Firstly, the location or the direction of the disturbance need to be clearly identified, only the fluctuation on the load side can be taken advantage of. Secondly, and thirdly, non-tirval changes of the voltage and current at the breaker are needed to calculate the parameters of the equivalent ciucuit with acceptable errors. The threshold value are needed to quantitively define the non-trivial change of the voltage or current. However, it is hard to find a once-forall settings as different power gird with a variaty of load type will have a diversity of characteristics. A fuzzy logic identifier is proposed to assess the disturbance in a more adaptive manner, which will be illustrated in the sequel.

\section{Design of fuzzy logic identifier}

The design of a fuzzy logic identifier usually includes three parts: (1) The rules or principles of the problem; (2) Selecting proper linguistic terms which can describe the input and output variables properly; (3) Defining fuzzy sets for all fuzzy variables [16-18].

\subsection{The Rules for Disturbance Identification}

As mentioned above, there are three variables need to be considered in fault level calculating process. Although the disturbance direction identification principle is clear as it directly compares Dir and zero, in practical application a margin need to be given. If Dir is very close to zero, it could lead to a mis-judgement as there exists errors both in PT and CT sensor as well as in the computation process. So the practical criterion is specified as:

$$
\left\{\begin{array}{l}
\text { Dir }<-\varepsilon, \text { grid side disturbance } \\
-\varepsilon<=\text { Dir }<=\varepsilon, \text { ignored } \\
\text { Dir }>\varepsilon, \text { load side disturbance }
\end{array}\right.
$$

where $\varepsilon$ is a predefined small positive value. Using this criterion, the unclear disturbance conditions, such as the disturbances simultaneously occurred on both sides, are ignored directly.

For the identification of the non-trivial voltage/current change, it is obvious that a relative value (percentage) will give a more reasonable result as for the same variation the one with larger voltage/current amplitude will be affected more slightly and vice versa. So the percentage of the voltage and current change relative to their average values are used for finding a non-trivial disturbance which are noted as follows:

$$
\begin{aligned}
& \Delta \widehat{\mathrm{U}}=\frac{|\Delta U|}{\operatorname{ave}\left(\left|\dot{U}_{1}\right|,\left|\dot{U}_{2}\right|\right)}=\frac{2\left|\dot{U}_{2}-\dot{U}_{1}\right|}{\left|\dot{U}_{1}\right|+\left|\dot{U}_{2}\right|} \\
& \Delta \hat{\mathrm{I}}=\frac{|\Delta I|}{\operatorname{ave}\left(\left|\dot{I}_{1}\right|,\left|\dot{I}_{2}\right|\right)}=\frac{2\left|\dot{I}_{2}-\dot{I}_{1}\right|}{\left|\dot{I}_{1}\right|+\left|\dot{I}_{2}\right|}
\end{aligned}
$$

It is obvious that these two positive parameters, range from 0 to 2, can be used for evaluating severity of the disturbance or the extent of the load fluctuation.

\subsection{Linguistic terms of the input-output variables}

It is an important step to represent the problem in fuzzy terms when fuzzy logic is used for problem-solving procedure. Four linguistic terms, "Large", "Medium", "Small" and "Zero" are picked out to describe the input and output variables; appending positive or negative sign to nonzero terms, totally 4 terms are used to describe input variables Dir, $\Delta U$ and $\Delta I$ in the fuzzy system [16]:

$$
\text { (O, PS, PM, PL) }
$$

$\mathrm{O} \quad-\quad$ Zero;

PS - Positive Small;

PM - Positive Medium;

$\mathrm{PL} \quad-\quad$ Positive Large.

Confidential level is the output variable, note as $C_{L}$, which represents the reliability of the disturbance and the final fault level result, seven terms of none negative value is required:

$$
\begin{array}{ll}
\text { (O, PVS, PS, PLM, PM, PML, PL) } \\
\mathrm{O} \quad-\quad \text { Zero; } \\
\text { PVS } \quad-\quad \text { Positive Very Small; }
\end{array}
$$




$\begin{array}{lll}\text { PS } & - & \text { Positive Small; } \\ \text { PLM } & - & \text { Positive Light Medium; } \\ \text { PM } & - & \text { Positive Medium; } \\ \text { PML } & & \text { Positive Medium Large; } \\ \text { PL } & - & \text { Positive Large. }\end{array}$

The input and output variables of a fuzzy logic identifier are usually specified within a range. But most of the time, the variation range of a variable can't be confirmed accurately. So a proper closed interval is needed, which the actual variation range can be projected to, for facilitating the calculation.

According to the feasible operation states and experiments, Dir can vary from $-\infty$ to $+\infty$, but will be mostly in the range of $[-10,10]$ and $\varepsilon$ can be defined as 0.001 ; For $\Delta U$, it normally varies in the range of $[0,0.3]$ as the voltage will not beyond the operating limitation; for $\Delta I$, it can be varied in a wide range of $[0,2]$ supposing the load can change in a wide range. In practical usage, only the conditions with positive Dir value are concerned, because the disturbance with a negative Dir will be ignored as they are identified to occur on the grid side.

The number of the elements in the basic universe should be more than twice of the total number of fuzzy linguistic terms to ensure that the fuzzy sets can cover the whole domain, and to avoid losing important information. Here, the universes of Dir, $\Delta U, \Delta I$, are chosen as:

$(0,1,2,3,4,5,6,7)$

The universe of the output variable $C_{L}$ are:

$$
(0,1,2,3,4,5,6,7,8,9,10,11,12,13)
$$

\subsection{Membership functions}

The values of the membership function are all in the interval $[0,1]$. 1 means that the object entirely belongs to the member of the set and 0 means it is totally irrelevant. When designing the fuzzy logic identifier, trapezoidal membership is an effective choice. The linguistic terms of input variables $\operatorname{Dir}, \Delta U, \Delta I$, the universes of them are all the same. Therefore, they have the same membership function (in Fig. 3). The membership function of the output $C_{L}$ is shown in Fig. 4.

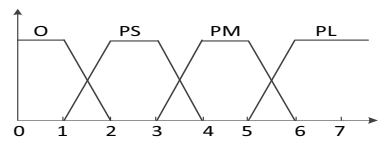

Figure 3. Membership functions of input variables.

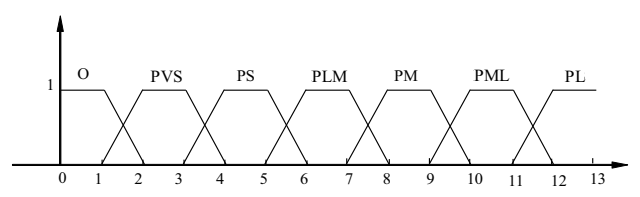

Figure 4. Membership function of output variables.

Taking advantage of the close relation between $\Delta U$ and $\Delta I$, we combine these two variables as one input signal as they change simultaneously. Consequently, the number of the fuzzy rules can be reduced to $4 * 4=16$, the detailed tuning rules are explicitly illustrated in Table I. So for different scenarios, the fault level results will have different confidential level.
Those of significant load change and clear positive Dir will be of high reliability.

Table 1.The fuzzy logic rules for forming the $C_{L}$

\begin{tabular}{|c|c|c|c|c|}
\hline${ }_{\Delta U I} D i r$ & $\mathrm{O}$ & PS & PM & PL \\
\hline $\mathrm{O}$ & $O$ & $P V S$ & $P V S$ & $P L M$ \\
\hline PS & $O$ & $P V S$ & PLM & $P M$ \\
\hline PM & $O$ & $P V S$ & $P M$ & $P M L$ \\
\hline PL & $O$ & $P L M$ & $P M L$ & $P L$ \\
\hline
\end{tabular}

\section{Simulation results}

The proposed scheme can be used in the protection relays without extra investment. If there is a fault on the feeder line, the relays will output trip signal in $100 \mathrm{~ms}$ and the FLP will be blocked at the same time because the fault condition will lead to serious errors as voltage distortion and the saturation of PT and CT. The practical implementation can be found in Fig. 5.The FLP also has a low voltage/current block function. If the voltage of the feeder is under $0.7 \mathrm{pu}$ with respect to the rating voltage, the FLP will be blocked to avoid giving a wrong result.

The phasor value of the positive voltage and current at the studied breaker is recorded and the Dir, $\Delta U$ and $\Delta I$ are calculated. The confidential level will be analyzed using the fuzzy rules in the fuzzy logic identifier. When the disturbance on the load side is detected, the fault level current value will be calculated. The output of the FLP module consists of the predicted fault level and the confidential level of the result. Only the local information is used for FLP and it can be directly realized in the relays without extra hardware investment. Though the fault current obtained is non-real time and is not exactly accurate. It is still helpful for the operators to know the change of the fault level as well as the potential danger so as to update the breakers or re-set the protection settings timely.

A distribution power grid (shown in Fig. 6) is used to test the effectiveness of the propose method. The topology of the model is established in PSCAD/EMTDC by referring to the typical complex distribution network with the integration of DGs. The fault level at breaker CB2 is studied and different types of load are connected to the feeder, including motors, electric arc furnace, constant resistance load and the composition of them.

The disturbance applied to the test system is as follows: 1) At $0 \mathrm{~s}$, all the main-sources are disconnected (Bil $\sim \mathrm{Bi} 6$ are open); 2) At $6 \mathrm{~s}$, half of the main-sources are connected (Bi1 $\sim \mathrm{Bi} 3$ closed); 3) 10s, all the other mainsources are connected (Bi4 $\mathrm{Bi} 6$ closed). The composition load changes randomly, the voltage and current at CB2

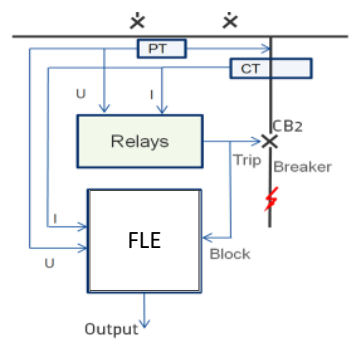

Figure 5. The practical implementation of the proposed scheme. 


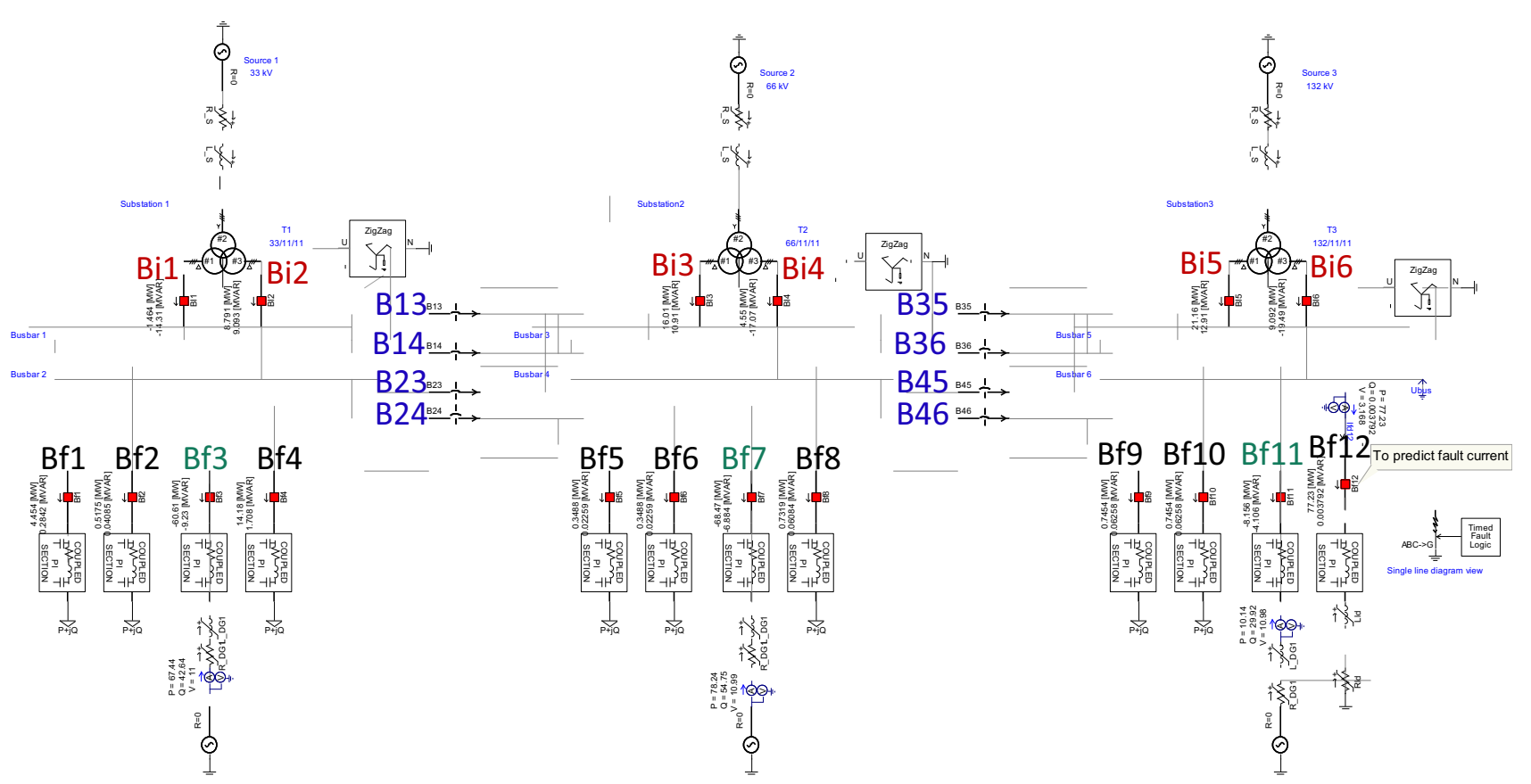

Figure 6. The test power system model in PSCAD/EMTDC.

$U / N$

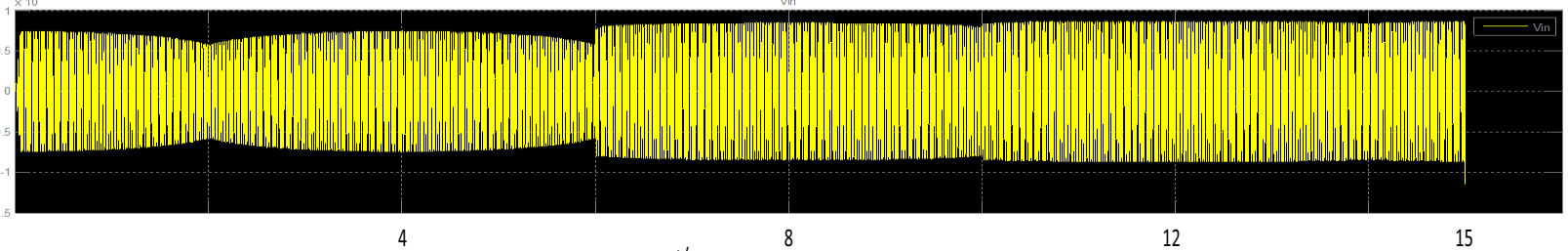

$\mathrm{t} / \mathrm{s}$

Figure 7. The instantaneous voltage at Bf12.

I/A

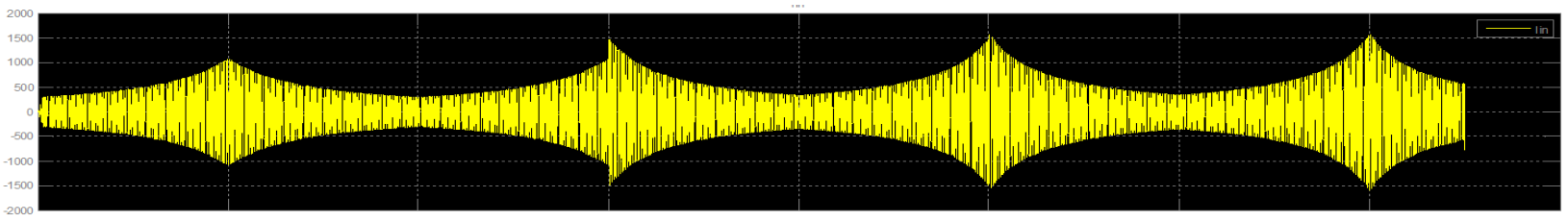

4

$\mathrm{t} / \mathrm{s}$

12

15

Figure 8. The instantaneous current at Bf12.

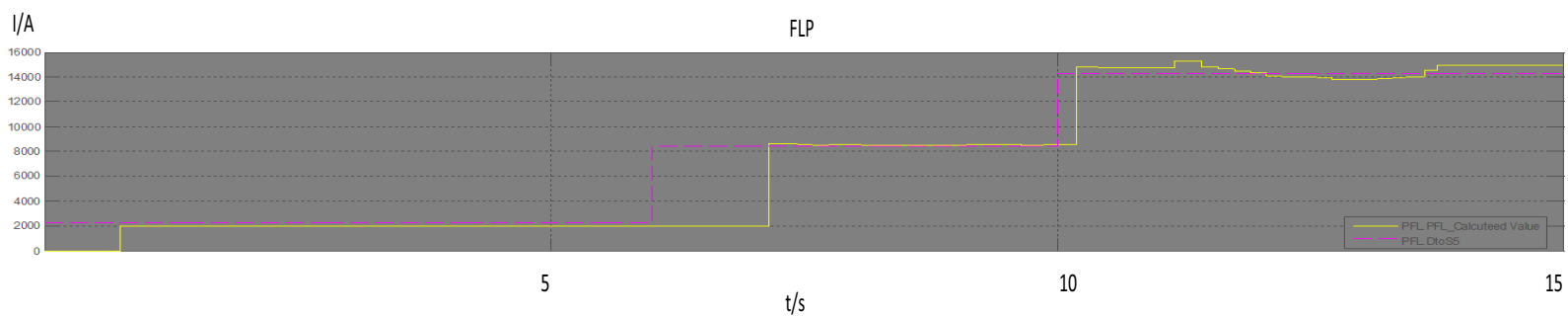

Figure 9. The actual fault current and the predicted fault current.

are shown in Fig. 7 and Fig. 8 respectively. The actual fault current (simulated using PSCAD) and the predicted fault current are shown in Fig. 9 with dashed line and solid line, respectively. Because the switching operation on the grid side, the actual fault current will change dramatically at $6 \mathrm{~s}$ and $10 \mathrm{~s}$, which is clearly shown with the purple dashed line. For the predicted fault current, because it needs to detect a non-trivial load fluctuation before the FLP working, there is a delay for its updating. But as soon as the fluctuation on the load side is detected, the predicted fault level will be given (as the solid line in 
Fig. 9) and it has a high similarity with the actual fault current value.

Eight other disturbances caused by motor power adjusting and electric arc furnace operating on the feeder are also tested using the proposed scheme. The maximum change of the voltage and current are different for each case. The dynamics of the voltage and current are not plotted here because of page limitation. The final statistic results are given in Table 2, in which the predicted fault current and the corresponding confidence level are given. It can be seen that the errors between the predicted fault current and the real value is no more than $5 \%$, which is accurate enough for the planning and for reminding the operators to upgrade the breaker or reset the protection relays settings. The case with more obvious voltage and current change will give out a more accurate result with a high confidence level.

Table 2. Test results for different scenarios.

\begin{tabular}{|c|c|c|c|}
\hline $\begin{array}{l}\text { Load } \\
\text { Type }\end{array}$ & $\max _{(\%)}(\Delta U I)$ & $\begin{array}{c}\text { Errors between } \\
\text { actual and predicted } \\
\text { fault } \\
\text { current }(\%)\end{array}$ & $\begin{array}{c}\text { Confidential } \\
\text { level }\end{array}$ \\
\hline \multirow{4}{*}{$\begin{array}{l}\text { Motor } \\
\text { load }\end{array}$} & $2 e-2$ & $4.46 \%$ & 4 \\
\hline & $3 e-2$ & $4.0 \%$ & 3 \\
\hline & $5 e-2$ & $3.07 \%$ & 5 \\
\hline & $10 \mathrm{e}-2$ & $2.82 \%$ & 7 \\
\hline \multirow{4}{*}{$\begin{array}{l}\text { electric } \\
\text { arc } \\
\text { furnace }\end{array}$} & $2 e-2$ & $5.0 \%$ & 3 \\
\hline & $3 e-2$ & $4.6 \%$ & 5 \\
\hline & $5 e-2$ & $3.66 \%$ & 6 \\
\hline & $10 \mathrm{e}-2$ & $2.58 \%$ & 8 \\
\hline
\end{tabular}

\section{Conclusion}

In this paper, a fault level predicting method is proposed using local measurement of the voltage and current. A fuzzy logic identifier is designed to adaptively rank the severity of the disturbance and to distinguish the direction of the disturbance in a distribution power system. The member function of the fuzzy logic identifier, which is essential to generate the confidential level of the final result, is also detailed illustrated. The implementation of the proposed scheme into a protective relay is introduced and a distribution power system is used to test the proposed method. The results show the effectiveness and accuracy of the presented scheme.

\section{Acknowledgement}

This work is supported by National Natural Science Foundation of China (No: 51407109), China Postdoctoral Science Foundation (2014M551904), and the Fundamental Research Funds of Shandong University (No. 2082014GN034).

\section{References}

1. G. Williamson, N. Jenkins, and G. Cornfield.Use of naturally occurring system disturbances to estimate the fault current contribution of induction motors.IEE Proceedings in Generation, Transmission and Distribution, 143, 3 (1996)
2. C. Mortley. The contribution to distribution network fault levels from the connection of distributed generation, KEMA Conference, London, UK, (2005)

3. S. Conner and A. Cruden.An automatic transient detection system which can be incorporated into an algorithm to accurately determine the fault level in networks with DG, International Universities Power Engineering Conference, London, UK, (2012)

4. H. Yazdanpanahi, Y. W. Li, and W. Xu. A new control strategy to mitigate the impact of inverterbased dgs on protection system. IEEE Transactions on Smart Grid, 3, 3(2012)

5. J. Berry, S. Jupe, M. Meisinger, and J. Outram. Implementation of an active fault level monitoring system for distributed generation integration.International Conference and Exhibition on Electricity Distribution , Stockholm, Sweden (2013)

6. P. Vovos and S. Hwachang. Analytical approach to large-scale system splitting for the regulation of fault levels. IEEE PowerTech Conference, Eindhoven, Holland, (2015)

7. M. S. Yahaya, M. F. Basar, Z. Ibrahim, M. N. N. Nasir, M. Y. Lada, and W. M. Bukhari. A Review on the Impact of Embedded Generation to Network Fault Level. International Conference on Mathematics Engineering and Industrial Applications,Penang, Malaysia (2014)

8. K. S. Ratnadeep, Y. N. Bhosale, and S. Kulkarni.Fault level analysis of power distribution system.Energy Efficient Technologies for Sustainability Conference (ICEETS), Nagercoil, India (2013)

9. E. S. Ibrahim and M. Idres. On-line fault level measurement. Electric Machines and Power Systems.26, 2(1998)

10. G. C. Cornfield.Estimating system fault level from naturally occurring disturbances. IEE Colloquium onFault Level Assessment Guessing with Greater Precision, London, UK (1996)

11. O. R. Ltd. Fault Level Prediction. [Online]. Available at: http://www.outramresearch.co.uk/flm/pages/product fault_level_overview.shtml

12. $\bar{H} . \mathrm{H}, \mathrm{S}$. Subramanian, and K. Venkataraman.A novel technique for fault level prediction in active distribution networks. Advanced Power System Automation and Protection Conference, Nanjing, China(2015)

13. E. S. IBRAHIM.Experimental Measurements of Fault Level For Sceco West, Kingdom of Saudi Arabia. Engineering Sciences, 15, 1 (2004)

14. M. R. Rylander, W. M. Grady, A. Mansoor, and F. Gorgette.Passive agent system impedance monitoring station and method.US Patents (2013)

15. G. Gurrala and I. Sen.Power system stabilizers design for interconnected power systems.IEEE Transactions on Power Systems, 25, 2 (2010)

16. H. Zhang, F. Shi, and Y. Liu.Enhancing optimal excitation control by adaptive fuzzy logic rules, International Journal of Electrical Power \& Energy Systems, 63, 1 (2014) 
17. J. M. Mendel, R. I. John, and F. Liu. Interval type-2 fuzzy logic systems made simple. IEEE Transactions on Fuzzy Systems, 14, 6(2006)
18. P. S. Bhati and R. Gupta. Robust fuzzy logic power system stabilizer based on evolution and learning. International Journal of Electrical Power \& Energy Systems, 53, 1 (2013) 